\title{
Coverage degree in mobile nodes wireless sensor network
}

\begin{abstract}
Coverage degree and mobility have acquired a lot of attention lately in Wireless Sensor Network (WSN). This paper shows that nodes mobility can enhance coverage degree for WSN. Analytical model is introduced to describe the coverage degree in mobile nodes wireless sensor network. MATLAB was used to build the simulator. The analytical work is validated by simulated results. Both of analytical model and simulation showing that the coverage degree has been enhanced in Mobile Nodes Wireless Sensor Network (MNWSN) as compared to static network.
\end{abstract}

Keyword: Coverage degree; Random way point model; Wireless sensor network 